\title{
A General Iterative Method of Fixed Points for Mixed Equilibrium Problems and Variational Inclusion Problems
}

\author{
Phayap Katchang ${ }^{1}$ and Poom Kumam ${ }^{1,2}$ \\ ${ }^{1}$ Department of Mathematics, Faculty of Science, King Mongkut's University of Technology Thonburi, \\ (KMUTT), Bangmod, Bangkok 10140, Thailand \\ ${ }^{2}$ Centre of Excellence in Mathematics, CHE, Si Ayutthaya Road, Bangkok 10400, Thailand \\ Correspondence should be addressed to Poom Kumam, poom.kum@kmutt.ac.th
}

Received 27 October 2009; Accepted 16 March 2010

Academic Editor: Jong Kyukkyungnam Kim

Copyright (c) 2010 P. Katchang and P. Kumam. This is an open access article distributed under the Creative Commons Attribution License, which permits unrestricted use, distribution, and reproduction in any medium, provided the original work is properly cited.

The purpose of this paper is to investigate the problem of finding a common element of the set of solutions for mixed equilibrium problems, the set of solutions of the variational inclusions with set-valued maximal monotone mappings and inverse-strongly monotone mappings, and the set of fixed points of a family of finitely nonexpansive mappings in the setting of Hilbert spaces. We propose a new iterative scheme for finding the common element of the above three sets. Our results improve and extend the corresponding results of the works by Zhang et al. (2008), Peng et al. (2008), Peng and Yao (2009), as well as Plubtieng and Sriprad (2009) and some well-known results in the literature.

\section{Introduction}

Throughout this paper, we assume that $H$ is a real Hilbert space with inner product and norm being denoted by $\langle\cdot, \cdot\rangle\rangle$ and $\|\cdot\|$, respectively, $2^{H}$ denoting the family of all subsets of $H$ and leting $C$ be a closed convex subset of $H$. A mapping $S: C \rightarrow C$ is called nonexpansive if $\|S x-S y\| \leq\|x-y\|$, for all $x, y \in C$. We use $F(S)$ to denote the set of fixed points of $S$, that is, $F(S)=\{x \in C: S x=x\}$. It is assumed throughout the paper that $S$ is a nonexpansive mapping such that $F(S) \neq \emptyset$. Recall that a self-mapping $f: C \rightarrow C$ is contraction on $C$ if there exists a constant $\alpha \in[0,1)$ and $x, y \in C$ such that $\|f(x)-f(y)\| \leq \alpha\|x-y\|$. Let $A$ be a strongly positive bounded linear operator on $H$ : that is, there is a constant $\bar{\gamma}>0$ with property

$$
\langle A x, x\rangle \geq \bar{\gamma}\|x\|^{2} \quad \forall x \in H .
$$


Let $A: H \rightarrow H$ be a single-valued nonlinear mapping and let $M: H \rightarrow 2^{H}$ be a set-valued mapping. We consider the following variational inclusion problem, which is to find a point $u \in H$ such that

$$
\theta \in A(u)+M(u)
$$

where $\theta$ is the zero vector in $H$. The set of solutions of problem (1.2) is denoted by $I(A, M)$.

If $M=\partial \phi: H \rightarrow 2^{H}$, where $\phi: H \rightarrow \mathbb{R} \cup\{+\infty\}$ is a proper convex lower semicontinuous function and $\partial \phi$ is the subdifferential of $\phi$, then the variational inclusion problem (1.2) is equivalent to find $u \in H$ such that

$$
\langle A u, v-u\rangle+\phi(y)-\phi(u) \geq 0, \quad \forall v, y \in H,
$$

which is called the mixed quasivariational inequality (see [1]).

If $M=\partial \delta_{C}$, where $C$ is a nonempty closed convex subset of $H$ and $\delta_{C}: H \rightarrow[0, \infty]$ is the indicator function of $C$, that is,

$$
\delta_{C}(x)= \begin{cases}0, & x \in C, \\ +\infty, & x \notin C,\end{cases}
$$

then the variational inclusion problem (1.2) is equivalent to find $u \in C$ such that

$$
\langle A u, v-u\rangle \geq 0, \quad \forall v \in H
$$

This problem is called Hartman-Stampacchia variational problem (see [2-4]).

A set-valued mapping $M: H \rightarrow 2^{H}$ is called monotone if, for all $x, y \in H, f \in M x$ and $g \in M y$ imply that $\langle x-y, f-g\rangle \geq 0$. A monotone mapping $M: H \rightarrow 2^{H}$ is maximal if the graph of $G(M)$ of $M$ is not properly contained in the graph of any other monotone mapping. It is known that a monotone mapping $M$ is maximal if and only if, for $(x, f) \in H \times H$, $\langle x-y, f-g\rangle \geq 0$ for every $(y, g) \in G(M)$ implies that $f \in M x$.

Let the set-valued mapping $M: H \rightarrow 2^{H}$ be a maximal monotone. We define the resolvent operator $J_{M, \lambda}$ that is associate with $M$ and $\lambda$ as follows:

$$
J_{M, \lambda}(u)=(I+\lambda M)^{-1}(u), \quad u \in H,
$$

where $\lambda$ is a positive number. It is worth mentioning that the resolvent operator $J_{M, \lambda}$ is singlevalued, nonexpansive, and 1-inverse-strongly monotone (see $[5,6]$ ).

Let $\varphi: C \rightarrow \mathbb{R} \cup\{+\infty\}$ be a proper extended real-valued function and let $F$ be a bifunction of $C \times C$ into $\mathbb{R}$, where $\mathbb{R}$ is the set of real numbers. Ceng and Yao [7] considered the following mixed equilibrium problem for finding $x \in C$ such that

$$
F(x, y)+\varphi(y) \geq \varphi(x) \quad \forall y \in C .
$$


The set of solutions of (1.7) is denoted by $\operatorname{MEP}(F, \varphi)$. We see that $x$ is a solution of problem (1.7) implying that $x \in \operatorname{dom} \varphi=\{x \in C \mid \varphi(x)<+\infty\}$. If $\varphi=0$, then the mixed equilibrium problem (1.7) becomes the following equilibrium problem that is to find $x \in C$ such that

$$
F(x, y) \geq 0 \quad \forall y \in C
$$

The set of solutions of (1.8) is denoted by $\mathrm{EP}(F)$. Given a mapping $T: C \rightarrow H$, let $F(x, y)=\langle T x, y-x\rangle$ for all $x, y \in C$. Then $z \in \operatorname{EP}(F)$ if and only if $\langle T z, y-z\rangle \geq 0$ for all $y \in C$, that is, $z$ is a solution of the variational inequality. The mixed equilibrium problems include fixed point problems, variational inequality problems, optimization problems, Nash equilibrium problems, and the equilibrium problem as special cases. Numerous problems in physics, optimization, and economics reduce to find a solution of (1.8). Some methods have been proposed to solve the equilibrium problem (see [8-21]).

In 2008, Zhang et al. [6] introduced an iterative scheme for finding a common element of the set of solutions to the variational inclusion problem with a multivalued maximal monotone mapping and an inverse-strongly monotone mapping and the set of fixed points of nonexpansive mapping in Hilbert spaces. The iterative scheme is $x_{0}=x \in H$, and:

$$
\begin{gathered}
y_{n}=J_{M, \lambda}\left(x_{n}-\lambda A x_{n}\right), \\
x_{n+1}=\alpha_{n} x+\left(1-\alpha_{n}\right) S y_{n}
\end{gathered}
$$

for all $n \geq 0$. They proved the strong convergence theorem under some mind conditions. In the same year, Peng et al. [22] introduced an iterative scheme by the viscosity approximate method for finding a common element of the set of solutions of a variational inclusion with set-valued maximal monotone mapping and inverse-strongly monotone mapping, the set of solutions of an equilibrium problem, and the set of fixed points of a nonexpansive mapping in Hilbert spaces. The sequence $\left\{x_{n}\right\}$ is generated as follows:

$$
\begin{gathered}
x_{1} \in H, \\
F\left(u_{n}, y\right)+\frac{1}{r_{n}}\left\langle y-u_{n}, u_{n}-x_{n}\right\rangle \geq 0, \quad \forall y \in C, \\
y_{n}=J_{M, \lambda}\left(u_{n}-\lambda A u_{n}\right), \\
x_{n+1}=\alpha_{n} f\left(x_{n}\right)+\left(1-\alpha_{n}\right) S y_{n}, \quad \forall n \in \mathbb{N} .
\end{gathered}
$$

They proved that if $\left\{\alpha_{n}\right\}$ and $\left\{r_{n}\right\}$ satisfy appropriate conditions, then $\left\{x_{n}\right\}$ converges strongly to $z \in F(S) \cap I(A, M)$, where $z=P_{F(S) \cap I(A, M)} f(z)$.

In 2009, Plubtieng and Sriprad [23] introduced an iterative method for finding a common element of the set of common fixed points of a countable family of nonexpansive mapping, the set of solutions of a variational inclusion with set-valued maximal monotone mapping and inverse-strongly monotone mappings, and the set of solutions of an equilibrium 
problem in Hilbert spaces. Starting with an arbitrary $x_{1} \in H$, define the sequences $\left\{x_{n}\right\},\left\{y_{n}\right\}$, and $\left\{u_{n}\right\}$ by

$$
\begin{gathered}
F\left(u_{n}, y\right)+\frac{1}{r_{n}}\left\langle y-u_{n}, u_{n}-x_{n}\right\rangle \geq 0, \quad \forall y \in C, \\
y_{n}=J_{M, \lambda}\left(u_{n}-\lambda A u_{n}\right), \\
x_{n+1}=\alpha_{n} \gamma f\left(x_{n}\right)+\left(1-\alpha_{n} B\right) S y_{n}, \quad \forall n \in \mathbb{N},
\end{gathered}
$$

where $A$ is an inverse-strongly monotone mapping and $B$ is a bounded linear operator on $H$. They proved that if the sequences $\left\{\alpha_{n}\right\}$ and $\left\{r_{n}\right\}$ of parameters satisfy appropriate conditions, then $\left\{x_{n}\right\}$ is generated by (1.11) converging strongly to the unique solution of the variational inequality

$$
\langle(B-\gamma f) z, z-x\rangle \leq 0, \quad \forall x \in F(S) \cap \operatorname{EP}(F) \cap I(A, M),
$$

which is the optimality condition for the minimization problem

$$
\left.\min _{x \in C} \frac{1}{2}\langle A x, x\rangle-h(x)\right\rangle \text {, }
$$

where $h$ is a potential function for $\gamma f$, that is, $h^{1}(x)=\gamma f(x)$, for all $x \in H$.

Let $T_{i}: C \rightarrow C$, where $i=1,2, \ldots, N$, be a family of finitely nonexpansive mappings. Let the mapping $W_{n}: C \rightarrow C$ be defined by

$$
\begin{gathered}
U_{n, 0}=I, \\
U_{n, 1}=\lambda_{n, 1} T_{1} U_{n, 0}+\left(1-\lambda_{n, 1}\right) I, \\
U_{n, 2}=\lambda_{n, 2} T_{2} U_{n, 1}+\left(1-\lambda_{n, 2}\right) I, \\
\vdots \\
U_{n, N-1}=\lambda_{n, N-1} T_{N-1} U_{n, N-2}+\left(1-\lambda_{n, N-1}\right) I, \\
W_{n}=U_{n, N}=\lambda_{n, N} T_{N} U_{n, N-1}+\left(1-\lambda_{n, N}\right) I,
\end{gathered}
$$

where $\left\{\lambda_{n, 1}\right\},\left\{\lambda_{n, 2}\right\}, \ldots,\left\{\lambda_{n, N}\right\} \in[0,1]$. Such a mapping $W_{n}$ is called the $W$-mapping generated by $T_{1}, T_{2}, \ldots, T_{N}$ and $\lambda_{n, 1}, \lambda_{n, 2}, \ldots, \lambda_{n, N}$. Nonexpansivity of each $T_{i}$ ensures the nonexpansivity of $W_{n}$. Moreover, in [24, Lemma 3.1], it is shown that $F\left(W_{n}\right)=\bigcap_{i=1}^{N} F\left(T_{i}\right)$.

The concept of $W$-mappings was introduced in $[25,26]$. It is now one of the main tools in studying convergence of iterative methods for approaching common fixed points of nonlinear mappings; more recent progresses can be found in $[24,27,28]$ and the references cited therein.

Following from $W$-mappings, Peng and Yao [29] introduced iterative schemes based on the extragradient method for finding a common element of the set of solutions of a generalized mixed equilibrium problem, the set of fixed points of a finite family of 
nonexpansive mappings, and the set of solutions of a variational inequality problem for a monotone, Lipschitz continuous mapping. The sequence $\left\{x_{n}\right\}$ is generated by

$$
\begin{gathered}
x_{1}=x \in C \\
F\left(u_{n}, y\right)+\varphi(y)-\varphi\left(u_{n}\right)+\left\langle B x_{n}, y-u_{n}\right\rangle+\frac{1}{r_{n}}\left\langle y-u_{n}, u_{n}-x_{n}\right\rangle \geq 0, \quad \forall y \in C \\
y_{n}=P_{C}\left(u_{n}-\lambda A u_{n}\right), \\
x_{n+1}=\alpha_{n} x_{n}+\left(1-\alpha_{n}\right) W_{n} P_{C}\left(u_{n}-\lambda A y_{n}\right), \quad \forall n \in \mathbb{N}
\end{gathered}
$$

where $A$ is monotone and Lipschitz continuous mapping and $B$ is an inverse-strongly monotone mapping. They proved the weak convergence theorem if the sequences $\left\{\alpha_{n}\right\}$ and $\left\{r_{n}\right\}$ of parameters satisfy appropriate conditions.

In this paper, motivated by the above results and the iterative schemes considered by Zhang et al. in [6], Peng et al. in [22], Peng and Yao in [29], and Plubtieng and Sriprad in [23], we present a new general iterative scheme for finding a common element of the set of solutions for mixed equilibrium problems, the set of solutions of the variational inclusions with set-valued maximal monotone mapping and inverse-strongly monotone mapping, and the set of fixed points of a family of finitely nonexpansive mappings in the setting of Hilbert spaces. Then, we prove strong convergence theorem under some mind conditions. Furthermore, by using above result, an iterative algorithm for solution of an optimization problem was obtained. The results presented in this paper extend and improve the results of Zhang et al. [6], Peng et al. [22], Peng and Yao [29], Plubtieng and Sriprad [23], and some authors.

\section{Preliminaries}

Let $H$ be a real Hilbert space with norm $\|\cdot\|$ and inner product $\langle\cdot, \cdot\rangle$ and let $C$ be a closed convex subset of $H$. When $\left\{x_{n}\right\}$ is a sequence in $H, x_{n} \rightarrow x$ means that $\left\{x_{n}\right\}$ converges weakly to $x$ and $x_{n} \rightarrow x$ means the strong convergence. In a real Hilbert space $H$, we have

$$
\begin{gathered}
\|x-y\|^{2}=\|x\|^{2}-\|y\|^{2}-2\langle x-y, y\rangle, \\
\|\lambda x+(1-\lambda) y\|^{2}=\lambda\|x\|^{2}+(1-\lambda)\|y\|^{2}-\lambda(1-\lambda)\|x-y\|^{2}
\end{gathered}
$$

for all $x, y \in H$ and $\lambda \in[0,1]$. For every point $x \in H$, there exists a unique nearest point in $C$, denoted by $P_{C} x$, such that

$$
\left\|x-P_{C} x\right\| \leq\|x-y\| \quad \forall y \in C .
$$

$P_{C}$ is called the metric projection of $H$ onto $C$. It is well known that $P_{C}$ is a nonexpansive mapping of $H$ onto $C$ and satisfies

$$
\left\langle x-y, P_{C} x-P_{C} y\right\rangle \geq\left\|P_{C} x-P_{C} y\right\|^{2}
$$


for every $x, y \in H$. Moreover, $P_{C} x$ is characterized by the following properties:

$$
\begin{gathered}
P_{C} x \in C, \\
\left\langle x-P_{C} x, y-P_{C} x\right\rangle \leq 0, \\
\|x-y\|^{2} \geq\left\|x-P_{C} x\right\|^{2}+\left\|y-P_{C} x\right\|^{2}
\end{gathered}
$$

for all $x \in H, y \in C$.

Recall that a mapping $A$ of $H$ into itself is called $\beta$-inverse-strongly monotone if there exists a positive real number $\beta$ such that

$$
\langle A u-A v, u-v\rangle \geq \alpha\|A u-A v\|^{2},
$$

for all $u, v \in H$. It is obvious that any $\beta$-inverse-strongly monotone mapping $A$ is $(1 / \beta)$ Lipschitz monotone and continuous mapping. We also have that, for all $u, v \in H$ and $\lambda>0$,

$$
\begin{aligned}
\|(I-\lambda A) u-(I-\lambda A) v\|^{2} & =\|(u-v)-\lambda(A u-A v)\|^{2} \\
& =\|u-v\|^{2}-2 \lambda\langle u-v, A u-A v\rangle+\lambda^{2}\|A u-A v\|^{2} \\
& \leq\|u-v\|^{2}+\lambda(\lambda-2 \alpha)\|A u-A v\|^{2} .
\end{aligned}
$$

So, if $\lambda \leq 2 \alpha$, then $I-\lambda A$ is a nonexpansive mapping from $H$ into itself.

It is also known that $H$ satisfies the Opial condition [30], that is, for any sequence $\left\{x_{n}\right\} \subset H$ with $x_{n} \rightarrow x$, the inequality

$$
\liminf _{n \rightarrow \infty}\left\|x_{n}-x\right\|<\liminf _{n \rightarrow \infty}\left\|x_{n}-y\right\|
$$

holds for every $y \in H$ with $x \neq y$.

For solving the mixed equilibrium problem, let us give the following assumptions for the bifunction $F, \varphi$, and the set $C$.

(A1) $F(x, x)=0$ for all $x \in C$.

(A2) $F$ is monotone, that is, $F(x, y)+F(y, x) \leq 0$ for all $x, y \in C$.

(A3) For each $x, y, z \in C, \lim _{t \rightarrow 0} F(t z+(1-t) x, y) \leq F(x, y)$.

(A4) For each $x \in C, y \mapsto F(x, y)$ is convex and lower semicontinuous.

(A5) For each $y \in C, x \mapsto F(x, y)$ is weakly upper semicontinuous.

(B1) For each $x \in H$ and $r>0$, there exist a bounded subset $D_{x} \subseteq C$ and $y_{x} \in C$, such that for any $z \in C \backslash D_{x}$,

$$
F\left(z, y_{x}\right)+\varphi\left(y_{x}\right)+\frac{1}{r}\left\langle y_{x}-z, z-x\right\rangle<\varphi(z)
$$

(B2) $C$ is a bounded set. 
We need the following lemmas for proving our main result.

Lemma 2.1 (Peng and Yao [31]). Let $C$ be a nonempty closed convex subset of $H$. Let $F: C \times C \rightarrow \mathbb{R}$ be a bifunction satisfying (A1)-(A5) and let $\varphi: C \rightarrow \mathbb{R} \cup\{+\infty\}$ be a proper lower semicontinuous and convex function. Assume that either (B1) or (B2) holds. For $r>0$ and $x \in H$, define a mapping $T_{r}: H \rightarrow C$ as follows:

$$
T_{r}(x)=\left\{z \in C: F(z, y)+\varphi(y)+\frac{1}{r}\langle y-z, z-x\rangle \geq \varphi(z), \forall y \in C\right\}
$$

for all $z \in H$. Then, the following hold.

(1) For each $x \in H, T_{r}(x) \neq \emptyset$.

(2) $T_{r}$ is single valued.

(3) $T_{r}$ is firmly nonexpansive, that is, for any $x, y \in H,\left\|T_{r} x-T_{r} y\right\|^{2} \leq\left\langle T_{r} x-T_{r} y, x-y\right\rangle$.

(4) $F\left(T_{r}\right)=\operatorname{MEP}(F, \varphi)$.

(5) $\operatorname{MEP}(F, \varphi)$ is closed and convex.

Lemma 2.2 (Xu [32]). Assume that $\left\{a_{n}\right\}$ is a sequence of nonnegative real numbers such that

$$
a_{n+1} \leq\left(1-\alpha_{n}\right) a_{n}+\delta_{n}, \quad n \geq 0,
$$

where $\left\{\alpha_{n}\right\}$ is a sequence in $(0,1)$ and $\left\{\delta_{n}\right\}$ is a sequence in $\mathbb{R}$ such that

(1) $\sum_{n=1}^{\infty} \alpha_{n}=\infty$,

(2) $\lim \sup _{n \rightarrow \infty}\left(\delta_{n} / \alpha_{n}\right) \leq 0$ or $\sum_{n=1}^{\infty}\left|\delta_{n}\right|<\infty$.

Then $\lim _{n \rightarrow \infty} a_{n}=0$.

Lemma 2.3 (Osilike and Igbokwe [33]). Let $(E,\langle\cdot, \cdot\rangle)$ be an inner product space. Then for all $x, y, z \in E$ and $\alpha, \beta, \gamma \in[0,1]$ with $\alpha+\beta+\gamma=1$, one has

$$
\|\alpha x+\beta y+\gamma z\|^{2}=\alpha\|x\|^{2}+\beta\|y\|^{2}+\gamma\|z\|^{2}-\alpha \beta\|x-y\|^{2}-\alpha \gamma\|x-z\|^{2}-\beta \gamma\|y-z\|^{2} .
$$

Lemma 2.4 (Colao et al. [28]). Let $C$ be a nonempty convex subset of a Banach space. Let $\left\{T_{i}\right\}_{i=1}^{N}$ be a family of finitely nonexpansive mappings of $C$ into itself and let $\left\{\lambda_{n, 1}\right\},\left\{\lambda_{n, 2}\right\}, \ldots,\left\{\lambda_{n, N}\right\}$ be sequences in $[0,1]$ such that $\lambda_{n, i} \rightarrow \lambda_{i}(i=1, \ldots, N)$. Moreover for every integer $n \geq 1$, let $W$ and $W_{n}$ be the $W$-mappings generated by $T_{1}, T_{2}, \ldots, T_{N}$ and $\lambda_{1}, \lambda_{2}, \ldots, \lambda_{N}$ and $T_{1}, T_{2}, \ldots, T_{N}$ and $\left\{\lambda_{n, 1}\right\},\left\{\lambda_{n, 2}\right\}, \ldots,\left\{\lambda_{n, N}\right\}$, respectively. Then for every $x \in C$, it follows that

$$
\lim _{n \rightarrow \infty}\left\|W_{n} x-W x\right\|=0
$$

Lemma 2.5 (Suzuki [34]). Let $\left\{x_{n}\right\}$ and $\left\{y_{n}\right\}$ be bounded sequences in a Banach space $X$ and let $\left\{\beta_{n}\right\}$ be a sequence in $[0,1]$ with $0<\liminf _{n \rightarrow \infty} \beta_{n} \leq \limsup _{n \rightarrow \infty} \beta_{n}<1$. Suppose that $x_{n+1}=$ $\left(1-\beta_{n}\right) y_{n}+\beta_{n} x_{n}$ for all integers $n \geq 0$ and $\lim \sup _{n \rightarrow \infty}\left(\left\|y_{n+1}-y_{n}\right\|-\left\|x_{n+1}-x_{n}\right\|\right) \leq 0$. Then, $\lim _{n \rightarrow \infty}\left\|y_{n}-x_{n}\right\|=0$. 
Lemma 2.6 (Marino and $\mathrm{Xu}[35]$ ). Assume that $A$ is a strongly positive linear bounded operator on a Hilbert space $H$ with coefficient $\bar{\gamma}>0$ and $0<\rho \leq\|A\|^{-1}$. Then $\|I-\rho A\| \leq 1-\rho \bar{\gamma}$.

Lemma 2.7 (Brézis [5]). Let $M: H \rightarrow 2^{H}$ be a maximal monotone mapping and $A: H \rightarrow H$ be a Lipschitz continuous mapping. Then the mapping $S=M+A: H \rightarrow 2^{H}$ is a maximal monotone mapping.

Remark 2.8. Lemma 2.7 implies that $I(A, M)$ is closed and convex if $M: H \rightarrow 2^{H}$ is a maximal monotone mapping and $A: H \rightarrow H$ is a Lipschitz continuous mapping.

Lemma 2.9 (Zhang et al. [6]). $u \in H$ is a solution of variational inclusion (1.2) if and only if $u=J_{M, \lambda}(u-\lambda A u)$, for all $\lambda>0$, that is,

$$
I(A, M)=F\left(J_{M, \lambda}(I-\lambda A)\right), \quad \forall \lambda>0 .
$$

\section{Main Result}

In this section, we prove a strong convergence theorem for finding a common element of the set of fixed points of a family of finitely nonexpansive mappings, the set of solutions of a mixed equilibrium problem, and the set of solutions of a variational inclusion problem for an inverse-strongly monotone mapping in a real Hilbert space.

Theorem 3.1. Let $C$ be a nonempty closed convex subset of a real Hilbert Space $H$. Let $F$ be a bifunction of $C \times C$ into real numbers $\mathbb{R}$ satisfying (A1)-(A5) and let $\varphi: C \rightarrow \mathbb{R} \cup\{+\infty\}$ be a proper lower semicontinuous and convex function. Let $f$ be a contraction of $H$ into itself with coefficient $\alpha \in(0,1)$. Let $A$ be an $\beta$-inverse-strongly monotone mapping of $H$ into itself, $M: H \rightarrow 2^{H}$ be a maximal monotone mapping, and let $B$ be a strongly bounded linear operator on $H$ with coefficient $\bar{\gamma}>0$ and $0<\gamma<\bar{\gamma} / \alpha$. Let $T_{1}, T_{2}, \ldots, T_{N}$ be a family of finitely nonexpansive mappings of $C$ into $H$ such that $\Omega:=\cap_{n=1}^{N} F\left(T_{i}\right) \cap I(A, M) \cap \operatorname{MEP}(F, \varphi) \neq \emptyset$ and let $W_{n}$ be the $W$-mapping generated by $T_{1}, T_{2}, \ldots, T_{N}$ and $\lambda_{n, 1}, \lambda_{n, 2}, \ldots, \lambda_{n, N}$. Assume that either (B1) or (B2) holds. Let $\left\{x_{n}\right\}$ be a sequence generated by $x_{1} \in H$ and

$$
\begin{gathered}
F\left(u_{n}, y\right)+\varphi(y)-\varphi\left(u_{n}\right)+\frac{1}{r_{n}}\left\langle y-u_{n}, u_{n}-x_{n}\right\rangle \geq 0, \quad \forall y \in C, \\
y_{n}=J_{M, \lambda}\left(u_{n}-\lambda A u_{n}\right), \\
v_{n}=J_{M, \lambda}\left(y_{n}-\lambda A y_{n}\right), \\
x_{n+1}=\alpha_{n} \gamma f\left(x_{n}\right)+\beta_{n} x_{n}+\left(\left(1-\beta_{n}\right) I-\alpha_{n} B\right) W_{n} v_{n}
\end{gathered}
$$

for every $n \geq 1$, where $\left\{\alpha_{n}\right\},\left\{\beta_{n}\right\} \subset(0,1),\left\{r_{n}\right\} \subset(0, \infty)$, and $\lambda \in(0,2 \beta)$ satisfy the following conditions:

(i) $\sum_{n=0}^{\infty} \alpha_{n}=\infty$ and $\lim _{n \rightarrow \infty} \alpha_{n}=0$,

(ii) $\liminf _{n \rightarrow \infty} r_{n}>0$ and $\lim _{n \rightarrow \infty}\left|r_{n+1}-r_{n}\right|=0$,

(iii) $0<\liminf _{n \rightarrow \infty} \beta_{n} \leq \limsup \sup _{n \rightarrow \infty} \beta_{n}<1$,

(iv) $\lim _{n \rightarrow \infty}\left|\lambda_{n, i}-\lambda_{n-1, i}\right|=0$ for all $i=1,2, \ldots, N$. 
Then $\left\{x_{n}\right\}$ converges strongly to $z \in \Omega$, where $z=P_{\Omega}(I-B+\gamma f)(z)$, which is the unique solution of the variational inequality

$$
\langle(B-\gamma f) z, z-x\rangle \leq 0, \quad x \in \Omega
$$

Proof. Since $\lim _{n \rightarrow \infty} \alpha_{n}=0$, we may assume, without loss of generality, that $\alpha_{n}<\left(1-\beta_{n}\right)\|B\|^{-1}$ for all $n$. We assume that $\|I-B\| \leq 1-\bar{\gamma}$. Since $B$ is linear bounded self-adjoint operator on $H$, we have

$$
\|B\|=\sup \{|\langle B x, x\rangle|: x \in H,\|x\|=1\} .
$$

Observe that

$$
\left\langle\left(\left(1-\beta_{n}\right) I-\alpha_{n} B\right) x, x\right\rangle=1-\beta_{n}-\alpha_{n}\langle B x, x\rangle \geq 1-\beta_{n}-\alpha_{n}\|B\| \geq 0,
$$

this shows that $\left(1-\beta_{n}\right) I-\alpha_{n} B$ is positive. It follows that

$$
\begin{aligned}
\left\|\left(1-\beta_{n}\right) I-\alpha_{n} B\right\| & =\sup \left\{\left|\left\langle\left(\left(1-\beta_{n}\right) I-\alpha_{n} B\right) x, x\right\rangle\right|: x \in H,\|x\|=1\right\} \\
& =\sup \left\{1-\beta_{n}-\alpha_{n}\langle B x, x\rangle: x \in H,\|x\|=1\right\} \\
& \leq 1-\beta_{n}-\alpha_{n} \bar{\gamma} .
\end{aligned}
$$

Let $p \in \Omega$, let $\left\{T_{r_{n}}\right\}$ be a sequence of mappings defined as in Lemma 2.1, and let $u_{n}=T_{r_{n}} x_{n}$. For any $n \in \mathbb{N}$, we have

$$
\left\|u_{n}-p\right\|=\left\|T_{r_{n}} x_{n}-T_{r_{n}} p\right\| \leq\left\|x_{n}-p\right\| .
$$

Since $p \in \Omega$, we have $p=J_{M, \lambda}(p-\lambda A p)$. From $J_{M, \lambda}$ and $I-\lambda A$ being nonexpansive, then we have

$$
\begin{aligned}
\left\|v_{n}-p\right\| & =\left\|J_{M, \lambda}\left(y_{n}-\lambda A y_{n}\right)-J_{M, \lambda}(p-\lambda A p)\right\| \\
& \leq\left\|\left(y_{n}-\lambda A y_{n}\right)-(p-\lambda A p)\right\| \\
& \leq\left\|y_{n}-p\right\| \\
& =\left\|J_{M, \lambda}\left(u_{n}-\lambda A u_{n}\right)-J_{M, \lambda}(p-\lambda A p)\right\| \\
& \leq\left\|\left(u_{n}-\lambda A u_{n}\right)-(p-\lambda A p)\right\| \\
& \leq\left\|u_{n}-p\right\| \\
& \leq\left\|x_{n}-p\right\|
\end{aligned}
$$


for all $n \in \mathbb{N}$. It follows that

$$
\begin{aligned}
\left\|x_{n+1}-p\right\| & =\left\|\alpha_{n} \gamma f\left(x_{n}\right)+\beta_{n} x_{n}+\left(\left(1-\beta_{n}\right) I-\alpha_{n} B\right) W_{n} v_{n}-p\right\| \\
& =\left\|\alpha_{n}\left(\gamma f\left(x_{n}\right)-B p\right)+\beta_{n}\left(x_{n}-p\right)+\left(\left(1-\beta_{n}\right) I-\alpha_{n} B\right)\left(W_{n} v_{n}-p\right)\right\| \\
& \leq \alpha_{n}\left\|\gamma f\left(x_{n}\right)-B p\right\|+\beta_{n}\left\|x_{n}-p\right\|+\left(1-\beta_{n}-\alpha_{n} \bar{\gamma}\right)\left\|v_{n}-p\right\| \\
& \leq \alpha_{n}\left\|\gamma f\left(x_{n}\right)-B p\right\|+\beta_{n}\left\|x_{n}-p\right\|+\left(1-\beta_{n}-\alpha_{n} \bar{\gamma}\right)\left\|x_{n}-p\right\| \\
& \leq \alpha_{n}\left\|\gamma f\left(x_{n}\right)-\gamma f(p)\right\|+\alpha_{n}\|\gamma f(p)-B p\|+\left(1-\alpha_{n} \bar{\gamma}\right)\left\|x_{n}-p\right\| \\
& \leq \alpha_{n} \gamma \alpha\left\|x_{n}-p\right\|+\alpha_{n}\|\gamma f(p)-B p\|+\left(1-\alpha_{n} \bar{\gamma}\right)\left\|x_{n}-p\right\| \\
& =\left(1-(\bar{\gamma}-\gamma \alpha) \alpha_{n}\right)\left\|x_{n}-p\right\|+(\bar{\gamma}-\gamma \alpha) \alpha_{n} \frac{\|\gamma f(p)-B p\|}{\bar{\gamma}-\gamma \alpha}
\end{aligned}
$$

for every $n \in \mathbb{N}$. It follows by mathematical induction that

$$
\left\|x_{n+1}-p\right\| \leq \max \left\{\left\|x_{1}-p\right\|, \frac{\|\gamma f(p)-B p\|}{\bar{\gamma}-\gamma \alpha}\right\}, \quad n \geq 1 .
$$

Therefore, $\left\{x_{n}\right\}$ is bounded. We also obtain that $\left\{u_{n}\right\},\left\{W_{n} v_{n}\right\},\left\{f\left(x_{n}\right)\right\},\left\{y_{n}\right\}$, and $\left\{v_{n}\right\}$, are all bounded.

Next, we show that $\lim _{n \rightarrow \infty}\left\|x_{n+1}-x_{n}\right\|=0$. Observing that $u_{n}=T_{r_{n}} x_{n} \in \operatorname{dom} \varphi$ and $u_{n+1}=T_{r_{n+1}} x_{n+1} \in \operatorname{dom} \varphi$, we get

$$
\begin{gathered}
F\left(u_{n}, y\right)+\varphi(y)-\varphi\left(u_{n}\right)+\frac{1}{r_{n}}\left\langle y-u_{n}, u_{n}-x_{n}\right\rangle \geq 0 \quad \forall y \in C, \\
F\left(u_{n+1}, y\right)+\varphi(y)-\varphi\left(u_{n+1}\right)+\frac{1}{r_{n+1}}\left\langle y-u_{n+1}, u_{n+1}-x_{n+1}\right\rangle \geq 0 \quad \forall y \in C .
\end{gathered}
$$

Take $y=u_{n+1}$ in (3.10) and $y=u_{n}$ in (3.11); by using condition (A2), we obtain

$$
\left\langle u_{n+1}-u_{n}, \frac{u_{n}-x_{n}}{r_{n}}-\frac{u_{n+1}-x_{n+1}}{r_{n+1}}\right\rangle \geq 0
$$

Thus $\left\langle u_{n+1}-u_{n}, u_{n}-u_{n+1}+x_{n+1}-x_{n}+\left(1-r_{n} / r_{n+1}\right)\left(u_{n+1}-x_{n+1}\right)\right\rangle \geq 0$. Without loss of generality, let us assume that there exists a real number $c$ such that $r_{n}>c$, for all $n \geq 1$. Then, we have

$$
\left\|u_{n+1}-u_{n}\right\|^{2} \leq\left\|u_{n+1}-u_{n}\right\|\left\{\left\|x_{n+1}-x_{n}\right\|+\left|1-\frac{r_{n}}{r_{n+1}}\right|\left\|u_{n+1}-x_{n+1}\right\|\right\}
$$


and hence

$$
\begin{aligned}
\left\|u_{n+1}-u_{n}\right\| & \leq\left\|x_{n+1}-x_{n}\right\|+\frac{1}{r_{n+1}}\left|r_{n+1}-r_{n}\right|\left\|u_{n+1}-x_{n+1}\right\| \\
& \leq\left\|x_{n+1}-x_{n}\right\|+\frac{1}{c}\left|r_{n+1}-r_{n}\right| M_{1}
\end{aligned}
$$

where $M_{1}=\sup \left\{\left\|u_{n}-x_{n}\right\|: n \in \mathbb{N}\right\}$.

On the other hand, again since $J_{M, \lambda}$ and $I-\lambda A$ are nonexpansive, we obtain

$$
\begin{aligned}
\left\|v_{n+1}-v_{n}\right\| & =\left\|J_{M, \lambda}\left(y_{n+1}-\lambda A y_{n+1}\right)-J_{M, \lambda}\left(y_{n}-\lambda A y_{n}\right)\right\| \\
& \leq\left\|\left(y_{n+1}-\lambda A y_{n+1}\right)-\left(y_{n}-\lambda A y_{n}\right)\right\| \\
& \leq\left\|y_{n+1}-y_{n}\right\| \\
& =\left\|J_{M, \lambda}\left(u_{n+1}-\lambda A u_{n+1}\right)-J_{M, \lambda}\left(u_{n}-\lambda A u_{n}\right)\right\| \\
& \leq\left\|\left(u_{n+1}-\lambda A u_{n+1}\right)-\left(u_{n}-\lambda A y_{n}\right)\right\| \\
& \leq\left\|u_{n+1}-u_{n}\right\| .
\end{aligned}
$$

It follows from (3.14) and (3.15) that

$$
\left\|v_{n+1}-v_{n}\right\| \leq\left\|x_{n+1}-x_{n}\right\|+\frac{1}{c}\left|r_{n+1}-r_{n}\right| M_{1}
$$

Define the sequence $\left\{z_{n}\right\}$ by $x_{n+1}=\left(1-\beta_{n}\right) z_{n}+\beta_{n} x_{n}$, for all $n \geq 1$. Then, observe that

$$
\begin{aligned}
z_{n} & =\frac{x_{n+1}-\beta_{n} x_{n}}{1-\beta_{n}} \\
& =\frac{\alpha_{n} \gamma f\left(x_{n}\right)+\beta_{n} x_{n}+\left(\left(1-\beta_{n}\right) I-\alpha_{n} B\right) W_{n} v_{n}-\beta_{n} x_{n}}{1-\beta_{n}} \\
& =\frac{\alpha_{n} \gamma f\left(x_{n}\right)+\left(\left(1-\beta_{n}\right) I-\alpha_{n} B\right) W_{n} v_{n}}{1-\beta_{n}} .
\end{aligned}
$$


It follows that

$$
\begin{aligned}
z_{n+1}-z_{n}= & \frac{\alpha_{n+1} \gamma f\left(x_{n+1}\right)+\left(\left(1-\beta_{n+1}\right) I-\alpha_{n+1} B\right) W_{n+1} v_{n+1}}{1-\beta_{n+1}} \\
& -\frac{\alpha_{n} \gamma f\left(x_{n}\right)+\left(\left(1-\beta_{n}\right) I-\alpha_{n} B\right) W_{n} v_{n}}{1-\beta_{n}} \\
= & \frac{\alpha_{n+1} \gamma f\left(x_{n+1}\right)}{1-\beta_{n+1}}+\frac{\left(1-\beta_{n+1}\right) W_{n+1} v_{n+1}}{1-\beta_{n+1}}-\frac{\alpha_{n+1} B W_{n+1} v_{n+1}}{1-\beta_{n+1}} \\
& -\frac{\alpha_{n} \gamma f\left(x_{n}\right)}{1-\beta_{n}}-\frac{\left(1-\beta_{n}\right) W_{n} v_{n}}{1-\beta_{n}}+\frac{\alpha_{n} B W_{n} v_{n}}{1-\beta_{n}} \\
= & \frac{\alpha_{n+1}}{1-\beta_{n+1}}\left(\gamma f\left(x_{n+1}\right)-B W_{n+1} v_{n+1}\right) \\
& +\frac{\alpha_{n}}{1-\beta_{n}}\left(B W_{n} v_{n}-\gamma f\left(x_{n}\right)\right)+W_{n+1} v_{n+1}-W_{n} v_{n} .
\end{aligned}
$$

From the definition of $W_{n}$, since $T_{i}$ and $U_{n, i}, i=1,2, \ldots, N$, are nonexpansive, we have

$$
\begin{aligned}
\left\|W_{n+1} v_{n}-W_{n} v_{n}\right\|= & \left\|\lambda_{n+1, N} T_{N} U_{n+1, N-1} v_{n}+\left(1-\lambda_{n+1, N}\right) v_{n}-\lambda_{n, N} T_{N} U_{n, N-1} v_{n}-\left(1-\lambda_{n, N}\right) v_{n}\right\| \\
\leq & \left|\lambda_{n+1, N}-\lambda_{n, N}\right|\left\|v_{n}\right\|+\left\|\lambda_{n+1, N} T_{N} U_{n+1, N-1} v_{n}-\lambda_{n, N} T_{N} U_{n, N-1} v_{n}\right\| \\
\leq & \left|\lambda_{n+1, N}-\lambda_{n, N}\right|\left\|v_{n}\right\|+\left\|\lambda_{n+1, N}\left(T_{N} U_{n+1, N-1} v_{n}-T_{N} U_{n, N-1} v_{n}\right)\right\| \\
& +\left|\lambda_{n+1, N}-\lambda_{n, N}\right|\left\|T_{N} U_{n, N-1} v_{n}\right\| \\
\leq & 2 M_{2}\left|\lambda_{n+1, N}-\lambda_{n, N}\right|+\lambda_{n+1, N}\left\|U_{n+1, N-1} v_{n}-U_{n, N-1} v_{n}\right\|,
\end{aligned}
$$

where $M_{2}$ is an approximate constant such that $M_{2} \geq \max \left\{\sup _{n \geq 1}\left\{\left\|v_{n}\right\|\right\}\right.$, $\left.\left\{\sup _{n \geq 1}\left\{\left\|T_{m} U_{n, m-1} v_{n}\right\|\right\} \mid m=1,2, \ldots, N\right\}\right\}$.

Since $0<\lambda_{n_{i}} \leq 1$ for all $n \geq 1$ and $i=1,2, \ldots, N$, we compute

$$
\begin{aligned}
&\left\|U_{n+1, N-1} v_{n}-U_{n, N-1} v_{n}\right\| \\
&=\left\|\lambda_{n+1, N-1} T_{N-1} U_{n+1, N-2} v_{n}+\left(1-\lambda_{n+1, N-1}\right) v_{n}-\lambda_{n, N-1} T_{N-1} U_{n, N-2} v_{n}-\left(1-\lambda_{n, N-1}\right) v_{n}\right\| \\
& \leq\left|\lambda_{n+1, N-1}-\lambda_{n, N-1}\right|\left\|v_{n}\right\|+\left\|\lambda_{n+1, N-1} T_{N-1} U_{n+1, N-2} v_{n}-\lambda_{n, N-1} T_{N-1} U_{n, N-2} v_{n}\right\| \\
& \leq\left|\lambda_{n+1, N-1}-\lambda_{n, N-1}\right|\left\|v_{n}\right\|+\left\|\lambda_{n+1, N-1}\left(T_{N-1} U_{n+1, N-2} v_{n}-T_{N-1} U_{n, N-2} v_{n}\right)\right\| \\
&+\left|\lambda_{n+1, N-1}-\lambda_{n, N-1}\right|\left\|T_{N-1} U_{n, N-2} v_{n}\right\| \\
& \leq 2 M_{2}\left|\lambda_{n+1, N-1}-\lambda_{n, N-1}\right|+\left\|U_{n+1, N-2} v_{n}-U_{n, N-2} v_{n}\right\| .
\end{aligned}
$$


It follows that

$$
\begin{aligned}
\left\|U_{n+1, N-1} v_{n}-U_{n, N-1} v_{n}\right\| \leq & 2 M_{2}\left|\lambda_{n+1, N-1}-\lambda_{n, N-1}\right|+2 M_{2}\left|\lambda_{n+1, N-2}-\lambda_{n, N-2}\right| \\
& +\left\|U_{n+1, N-3} v_{n}-U_{n, N-3} v_{n}\right\| \\
\leq & 2 M_{2} \sum_{i=2}^{N-1}\left|\lambda_{n+1, i}-\lambda_{n, i}\right|+\left\|U_{n+1,1} v_{n}-U_{n, 1} v_{n}\right\| \\
= & 2 M_{2} \sum_{i=2}^{N-1}\left|\lambda_{n+1, i}-\lambda_{n, i}\right| \\
& +\left\|\lambda_{n+1,1} T_{1} v_{n}+\left(1-\lambda_{n+1,1}\right) v_{n}-\lambda_{n, 1} T_{1} v_{n}-\left(1-\lambda_{n, 1}\right) v_{n}\right\| \\
\leq & 2 M_{2} \sum_{i=1}^{N-1}\left|\lambda_{n+1, i}-\lambda_{n, i}\right| .
\end{aligned}
$$

Substituting (3.21) into (3.19) yields that

$$
\begin{aligned}
\left\|W_{n+1} v_{n}-W_{n} v_{n}\right\| & \leq 2 M_{2}\left|\lambda_{n+1, N}-\lambda_{n, N}\right|+2 \lambda_{n+1, N} M_{2} \sum_{i=1}^{N-1}\left|\lambda_{n+1, i}-\lambda_{n, i}\right| \\
& \leq 2 M_{2} \sum_{i=1}^{N}\left|\lambda_{n+1, i}-\lambda_{n, i}\right|
\end{aligned}
$$

and hence

$$
\begin{aligned}
\left\|W_{n+1} v_{n+1}-W_{n} v_{n}\right\| & \leq\left\|W_{n+1} v_{n+1}-W_{n+1} v_{n}\right\|+\left\|W_{n+1} v_{n}-W_{n} v_{n}\right\| \\
& \leq\left\|v_{n+1}-v_{n}\right\|+2 M_{2} \sum_{i=1}^{N}\left|\lambda_{n+1, i}-\lambda_{n, i}\right| .
\end{aligned}
$$

Combining (3.16) and (3.23), we obtain

$$
\begin{aligned}
\left\|z_{n+1}-z_{n}\right\| \leq & \frac{\alpha_{n+1}}{1-\beta_{n+1}}\left(\left\|\gamma f\left(x_{n+1}\right)\right\|+\left\|B W_{n+1} v_{n+1}\right\|\right)+\frac{\alpha_{n}}{1-\beta_{n}}\left(\left\|B W_{n} v_{n}\right\|+\left\|\gamma f\left(x_{n}\right)\right\|\right) \\
& +\left\|W_{n+1} v_{n+1}-W_{n} v_{n}\right\| \\
\leq & \frac{\alpha_{n+1}}{1-\beta_{n+1}}\left(\left\|\gamma f\left(x_{n+1}\right)\right\|+\left\|B W_{n+1} v_{n+1}\right\|\right)+\frac{\alpha_{n}}{1-\beta_{n}}\left(\left\|B W_{n} v_{n}\right\|+\left\|\gamma f\left(x_{n}\right)\right\|\right) \\
& +\left\|v_{n+1}-v_{n}\right\|+2 M_{2} \sum_{i=1}^{N}\left|\lambda_{n+1, i}-\lambda_{n, i}\right| \\
\leq & \frac{\alpha_{n+1}}{1-\beta_{n+1}}\left(\left\|\gamma f\left(x_{n+1}\right)\right\|+\left\|B W_{n+1} v_{n+1}\right\|\right)+\frac{\alpha_{n}}{1-\beta_{n}}\left(\left\|B W_{n} v_{n}\right\|+\left\|\gamma f\left(x_{n}\right)\right\|\right) \\
& +\left\|x_{n+1}-x_{n}\right\|+\frac{1}{c}\left|r_{n+1}-r_{n}\right| M_{1}+2 M_{2} \sum_{i=1}^{N}\left|\lambda_{n+1, i}-\lambda_{n, i}\right| .
\end{aligned}
$$


So

$$
\begin{aligned}
\left\|z_{n+1}-z_{n}\right\|-\left\|x_{n+1}-x_{n}\right\| \leq & \frac{\alpha_{n+1}}{1-\beta_{n+1}}\left(\left\|\gamma f\left(x_{n+1}\right)\right\|+\left\|B W_{n+1} v_{n+1}\right\|\right) \\
& +\frac{\alpha_{n}}{1-\beta_{n}}\left(\left\|B W_{n} v_{n}\right\|+\left\|\gamma f\left(x_{n}\right)\right\|\right) \\
& +\frac{1}{c}\left|r_{n+1}-r_{n}\right| M_{1}+2 M_{2} \sum_{i=1}^{N}\left|\lambda_{n+1, i}-\lambda_{n, i}\right| .
\end{aligned}
$$

Conditions (i)-(iv) imply that

$$
\limsup _{n \rightarrow \infty}\left(\left\|z_{n+1}-z_{n}\right\|-\left\|x_{n+1}-x_{n}\right\|\right) \leq 0
$$

Hence, by Lemma 2.5, we have

$$
\lim _{n \rightarrow \infty}\left\|z_{n}-x_{n}\right\|=0
$$

Consequently,

$$
\lim _{n \rightarrow \infty}\left\|x_{n+1}-x_{n}\right\|=\lim _{n \rightarrow \infty}\left(1-\beta_{n}\right)\left\|z_{n}-x_{n}\right\|=0 .
$$

From (ii), (3.14), (3.16), and (3.28), we also have $\left\|u_{n+1}-u_{n}\right\| \rightarrow 0$ and $\left\|v_{n+1}-v_{n}\right\| \rightarrow 0$ as $n \rightarrow \infty$. We note that

$$
\begin{aligned}
x_{n+1}-x_{n}= & \alpha_{n} \gamma f\left(x_{n}\right)+\beta_{n} x_{n}+\left(\left(1-\beta_{n}\right) I-\alpha_{n} B\right) W_{n} v_{n}-x_{n} \\
= & \alpha_{n} \gamma f\left(x_{n}\right)-\alpha_{n} B x_{n}+\alpha_{n} B x_{n}+\beta_{n} x_{n}+\left(\left(1-\beta_{n}\right) I-\alpha_{n} B\right) W_{n} v_{n} \\
& -\left(\left(1-\beta_{n}\right) I-\alpha_{n} B\right) x_{n}+\left(\left(1-\beta_{n}\right) I-\alpha_{n} B\right) x_{n}-x_{n} \\
= & \alpha_{n}\left(\gamma f\left(x_{n}\right)-B x_{n}\right)+\left(\left(1-\beta_{n}\right) I-\alpha_{n} B\right)\left(W_{n} v_{n}-x_{n}\right) \\
\leq & \alpha_{n}\left(\gamma f\left(x_{n}\right)-B x_{n}\right)+\left(1-\beta_{n}-\alpha_{n} \bar{\gamma}\right)\left(W_{n} v_{n}-x_{n}\right)
\end{aligned}
$$

It follows that

$$
\left(1-\beta_{n}-\alpha_{n} \bar{\gamma}\right)\left\|x_{n}-W_{n} v_{n}\right\| \leq \alpha_{n}\left\|\gamma f\left(x_{n}\right)-B x_{n}\right\|+\left\|x_{n}-x_{n+1}\right\|,
$$


From (i), (iii), and (3.28), we obtain

$$
\lim _{n \rightarrow \infty}\left\|x_{n}-W_{n} v_{n}\right\|=0
$$

Next, we shall show that $\lim _{n \rightarrow \infty}\left\|x_{n}-u_{n}\right\|=0$. For any $p \in \Omega$, since $T_{r_{n}}$ is firmly nonexpansive, we have

$$
\begin{aligned}
\left\|u_{n}-p\right\|^{2} & =\left\|T_{r_{n}} x_{n}-T_{r_{n}} p\right\|^{2} \leq\left\langle T_{r_{n}} x_{n}-T_{r_{n}} p, x_{n}-p\right\rangle=\left\langle u_{n}-p, x_{n}-p\right\rangle \\
& =\frac{1}{2}\left(\left\|u_{n}-p\right\|^{2}+\left\|x_{n}-p\right\|^{2}-\left\|u_{n}-x_{n}\right\|^{2}\right) .
\end{aligned}
$$

It follows that

$$
\left\|u_{n}-p\right\|^{2} \leq\left\|x_{n}-p\right\|^{2}-\left\|x_{n}-u_{n}\right\|^{2} .
$$

Therefore, we have

$$
\begin{aligned}
\left\|x_{n+1}-p\right\|^{2} & =\left\|\alpha_{n} \gamma f\left(x_{n}\right)+\beta_{n} x_{n}+\left(\left(1-\beta_{n}\right) I-\alpha_{n} B\right) W_{n} v_{n}-p\right\|^{2} \\
& =\left\|\alpha_{n}\left(\gamma f\left(x_{n}\right)-B p\right)+\beta_{n}\left(x_{n}-p\right)+\left(\left(1-\beta_{n}\right) I-\alpha_{n} B\right)\left(W_{n} v_{n}-p\right)\right\|^{2} \\
& \leq \alpha_{n}\left\|\gamma f\left(x_{n}\right)-B p\right\|^{2}+\beta_{n}\left\|x_{n}-p\right\|^{2}+\left(1-\beta_{n}-\alpha_{n} \bar{\gamma}\right)\left\|v_{n}-p\right\|^{2} \\
& \leq \alpha_{n}\left\|\gamma f\left(x_{n}\right)-B p\right\|^{2}+\beta_{n}\left\|x_{n}-p\right\|^{2}+\left(1-\beta_{n}-\alpha_{n} \bar{\gamma}\right)\left\|u_{n}-p\right\|^{2} \\
& \leq \alpha_{n}\left\|\gamma f\left(x_{n}\right)-B p\right\|^{2}+\beta_{n}\left\|x_{n}-p\right\|^{2}+\left(1-\beta_{n}-\alpha_{n} \bar{\gamma}\right)\left(\left\|x_{n}-p\right\|^{2}-\left\|x_{n}-u_{n}\right\|^{2}\right) \\
& =\alpha_{n}\left\|\gamma f\left(x_{n}\right)-B p\right\|^{2}+\left(1-\alpha_{n} \bar{\gamma}\right)\left\|x_{n}-p\right\|^{2}-\left(1-\beta_{n}-\alpha_{n} \bar{\gamma}\right)\left\|x_{n}-u_{n}\right\|^{2} .
\end{aligned}
$$

Then, we obtain

$$
\begin{aligned}
\left(1-\beta_{n}-\alpha_{n} \bar{\gamma}\right)\left\|x_{n}-u_{n}\right\|^{2} & \leq \alpha_{n}\left\|\gamma f\left(x_{n}\right)-B p\right\|^{2}+\left(1-\alpha_{n} \bar{\gamma}\right)\left\|x_{n}-p\right\|^{2}-\left\|x_{n+1}-p\right\|^{2} \\
& \leq \alpha_{n}\left\|\gamma f\left(x_{n}\right)-B p\right\|^{2}+\left\|x_{n+1}-x_{n}\right\|\left(\left\|x_{n}-p\right\|+\left\|x_{n+1}-p\right\|\right) .
\end{aligned}
$$


By (i), (iii), and (3.28) imply that

$$
\lim _{n \rightarrow \infty}\left\|x_{n}-u_{n}\right\|=0
$$

Since $\liminf _{n \rightarrow \infty} r_{n}>0$, we have

$$
\lim _{n \rightarrow \infty}\left\|\frac{x_{n}-u_{n}}{r_{n}}\right\|=\lim _{n \rightarrow \infty} \frac{1}{r_{n}}\left\|x_{n}-u_{n}\right\|=0 .
$$

We note that, by (3.34), nonexpansiveness of $J_{M, \lambda}$ and the inverse-strong monotonicity of $A$ imply that

$$
\begin{aligned}
\left\|x_{n+1}-p\right\|^{2} \leq & \alpha_{n}\left\|\gamma f\left(x_{n}\right)-B p\right\|^{2}+\beta_{n}\left\|x_{n}-p\right\|^{2}+\left(1-\beta_{n}-\alpha_{n} \bar{\gamma}\right)\left\|v_{n}-p\right\|^{2} \\
= & \alpha_{n}\left\|\gamma f\left(x_{n}\right)-B p\right\|^{2}+\beta_{n}\left\|x_{n}-p\right\|^{2} \\
& +\left(1-\beta_{n}-\alpha_{n} \bar{\gamma}\right)\left\|J_{M, \lambda}\left(y_{n}-\lambda A y_{n}\right)-J_{M, \lambda}(p-\lambda A p)\right\|^{2} \\
\leq & \alpha_{n}\left\|\gamma f\left(x_{n}\right)-B p\right\|^{2}+\beta_{n}\left\|x_{n}-p\right\|^{2} \\
& +\left(1-\beta_{n}-\alpha_{n} \bar{\gamma}\right)\left\|\left(y_{n}-\lambda A y_{n}\right)-(p-\lambda A p)\right\| \\
\leq & \alpha_{n}\left\|\gamma f\left(x_{n}\right)-B p\right\|^{2}+\beta_{n}\left\|x_{n}-p\right\|^{2} \\
& +\left(1-\beta_{n}-\alpha_{n} \bar{\gamma}\right)\left\{\left\|y_{n}-p\right\|^{2}+\lambda(\lambda-2 \beta)\left\|A y_{n}-A p\right\|^{2}\right\} \\
\leq & \alpha_{n}\left\|\gamma f\left(x_{n}\right)-B p\right\|^{2}+\beta_{n}\left\|x_{n}-p\right\|^{2} \\
& +\left(1-\beta_{n}-\alpha_{n} \bar{\gamma}\right)\left\|x_{n}-p\right\|^{2}+\left(1-\beta_{n}-\alpha_{n} \bar{\gamma}\right) \lambda(\lambda-2 \beta)\left\|A y_{n}-A p\right\|^{2} \\
\leq & \alpha_{n}\left\|\gamma f\left(x_{n}\right)-B p\right\|^{2}+\left\|x_{n}-p\right\|^{2}+\left(1-\beta_{n}-\alpha_{n} \bar{\gamma}\right) \lambda(\lambda-2 \beta)\left\|A y_{n}-A p\right\|^{2} .
\end{aligned}
$$

It follows from (i), (iii), and (3.28) that

$$
\begin{aligned}
0 & \leq\left(1-\beta_{n}-\alpha_{n} \bar{\gamma}\right) \lambda(2 \beta-\lambda)\left\|A y_{n}-A p\right\|^{2} \\
& \leq \alpha_{n}\left\|\gamma f\left(x_{n}\right)-B p\right\|^{2}+\left\|x_{n}-p\right\|^{2}-\left\|x_{n+1}-p\right\|^{2} \\
& \leq \alpha_{n}\left\|\gamma f\left(x_{n}\right)-B p\right\|^{2}+\left\|x_{n}-x_{n+1}\right\|\left(\left\|x_{n}-p\right\|+\left\|x_{n+1}-p\right\|\right) \longrightarrow 0,
\end{aligned}
$$

which implies that

$$
\left\|A y_{n}-A p\right\| \longrightarrow 0, \quad \text { as } n \longrightarrow \infty
$$


On the other hand, since $J_{M, \lambda}$ is firmly nonexpansive, we have

$$
\begin{aligned}
\left\|v_{n}-p\right\|^{2}= & \left\|J_{M, \lambda}\left(y_{n}-\lambda A y_{n}\right)-J_{M, \lambda}(p-\lambda A p)\right\|^{2} \\
\leq & \left\langle\left(y_{n}-\lambda A y_{n}\right)-(p-\lambda A p), v_{n}-p\right\rangle \\
= & \frac{1}{2}\left\{\left\|\left(y_{n}-\lambda A y_{n}\right)-(p-\lambda A p)\right\|^{2}+\left\|v_{n}-p\right\|^{2}\right. \\
& \left.-\left\|\left(y_{n}-\lambda A y_{n}\right)-(p-\lambda A p)-\left(v_{n}-p\right)\right\|^{2}\right\} \\
\leq & \frac{1}{2}\left\{\left\|y_{n}-p\right\|^{2}+\left\|v_{n}-p\right\|^{2}-\left\|\left(y_{n}-v_{n}\right)-\lambda\left(A y_{n}-A p\right)\right\|^{2}\right\} \\
= & \frac{1}{2}\left\{\left\|y_{n}-p\right\|^{2}+\left\|v_{n}-p\right\|^{2}-\left\|y_{n}-v_{n}\right\|^{2}\right. \\
& \left.+2 \lambda\left\langle y_{n}-v_{n}, A y_{n}-A p\right\rangle-\lambda^{2}\left\|A y_{n}-A p\right\|^{2}\right\} \\
\leq & \frac{1}{2}\left\{\left\|y_{n}-p\right\|^{2}+\left\|v_{n}-p\right\|^{2}-\left\|y_{n}-v_{n}\right\|^{2}\right. \\
& \left.+2 \lambda\left\|y_{n}-v_{n}\right\|\left\|A y_{n}-A p\right\|-\lambda^{2}\left\|A y_{n}-A p\right\|^{2}\right\},
\end{aligned}
$$

which yields that

$$
\left\|v_{n}-p\right\|^{2} \leq\left\|y_{n}-p\right\|^{2}-\left\|y_{n}-v_{n}\right\|^{2}+2 \lambda\left\|y_{n}-v_{n}\right\|\left\|A y_{n}-A p\right\|
$$

From (3.34) and (3.42), we obtain

$$
\begin{aligned}
\left\|x_{n+1}-p\right\|^{2} \leq & \alpha_{n}\left\|\gamma f\left(x_{n}\right)-B p\right\|^{2}+\beta_{n}\left\|x_{n}-p\right\|^{2}+\left(1-\beta_{n}-\alpha_{n} \bar{\gamma}\right)\left\|v_{n}-p\right\|^{2} \\
\leq & \alpha_{n}\left\|\gamma f\left(x_{n}\right)-B p\right\|^{2}+\beta_{n}\left\|x_{n}-p\right\|^{2} \\
& +\left(1-\beta_{n}-\alpha_{n} \bar{\gamma}\right)\left\{\left\|y_{n}-p\right\|^{2}-\left\|y_{n}-v_{n}\right\|^{2}+2 \lambda\left\|y_{n}-v_{n}\right\|\left\|A y_{n}-A p\right\|\right\} \\
\leq & \alpha_{n}\left\|\gamma f\left(x_{n}\right)-B p\right\|^{2}+\beta_{n}\left\|x_{n}-p\right\|^{2}+\left(1-\beta_{n}-\alpha_{n} \bar{\gamma}\right)\left\|x_{n}-p\right\|^{2} \\
& -\left(1-\beta_{n}-\alpha_{n} \bar{\gamma}\right)\left\|y_{n}-v_{n}\right\|^{2} \\
& +2 \lambda\left(1-\beta_{n}-\alpha_{n} \bar{\gamma}\right)\left\|y_{n}-v_{n}\right\|\left\|A y_{n}-A p\right\| \\
\leq & \alpha_{n}\left\|\gamma f\left(x_{n}\right)-B p\right\|^{2}+\left\|x_{n}-p\right\|^{2}-\left(1-\beta_{n}-\alpha_{n} \bar{\gamma}\right)\left\|y_{n}-v_{n}\right\|^{2} \\
& +2 \lambda\left(1-\beta_{n}-\alpha_{n} \bar{\gamma}\right)\left\|y_{n}-v_{n}\right\|\left\|A y_{n}-A p\right\| .
\end{aligned}
$$


Hence, we get

$$
\begin{aligned}
\left(1-\beta_{n}-\alpha_{n} \bar{\gamma}\right)\left\|y_{n}-v_{n}\right\|^{2} \leq & \alpha_{n}\left\|\gamma f\left(x_{n}\right)-B p\right\|^{2}+\left\|x_{n}-p\right\|^{2}-\left\|x_{n+1}-p\right\|^{2} \\
& +2 \lambda\left(1-\beta_{n}-\alpha_{n} \bar{\gamma}\right)\left\|y_{n}-v_{n}\right\|\left\|A y_{n}-A p\right\| \\
\leq & \alpha_{n}\left\|\gamma f\left(x_{n}\right)-B p\right\|^{2}+\left\|x_{n}-x_{n+1}\right\|\left(\left\|x_{n}-p\right\|+\left\|x_{n+1}-p\right\|\right) \\
& +2 \lambda\left(1-\beta_{n}-\alpha_{n} \bar{\gamma}\right)\left\|y_{n}-v_{n}\right\|\left\|A y_{n}-A p\right\| .
\end{aligned}
$$

By (i), (iii), (3.28), and (3.40), we have

$$
\lim _{n \rightarrow \infty}\left\|y_{n}-v_{n}\right\|=0
$$

Similarly, we can prove that

$$
\lim _{n \rightarrow \infty}\left\|A u_{n}-A p\right\|=0
$$

By the same idea in (3.42), (3.45) and using (3.46), then we obtain that

$$
\lim _{n \rightarrow \infty}\left\|u_{n}-y_{n}\right\|=0
$$

From

$$
\left\|W_{n} v_{n}-v_{n}\right\| \leq\left\|W_{n} v_{n}-x_{n}\right\|+\left\|x_{n}-u_{n}\right\|+\left\|u_{n}-y_{n}\right\|+\left\|y_{n}-v_{n}\right\|,
$$

hence

$$
\lim _{n \rightarrow \infty}\left\|W_{n} v_{n}-v_{n}\right\|=0,
$$

and also

$$
\left\|v_{n}-x_{n}\right\| \leq\left\|v_{n}-y_{n}\right\|+\left\|y_{n}-u_{n}\right\|+\left\|u_{n}-x_{n}\right\| \longrightarrow 0 \quad \text { as } n \longrightarrow \infty
$$

Observe that $P_{\Omega}(I-B+\gamma f)$ is a contraction of $H$ into itself. Indeed, for all $x, y \in H$, we have

$$
\begin{aligned}
\left\|P_{\Omega}(I-B+\gamma f)(x)-P_{\Omega}(I-B+\gamma f)(y)\right\| & \leq\|(I-B+\gamma f)(x)-(I-B+\gamma f)(y)\| \\
& \leq\|I-B\|\|x-y\|+\gamma\|f(x)-f(y)\| \\
& \leq(1-\bar{\gamma})\|x-y\|+\gamma \alpha\|x-y\| \\
& =(1-(\bar{\gamma}-\gamma \alpha))\|x-y\| .
\end{aligned}
$$


Since $H$ is complete, there exists a unique fixed point $z \in H$ such that $z=P_{\Omega}(I-B+\gamma f) z$. Next, we show that

$$
\limsup _{n \rightarrow \infty}\left\langle(B-\gamma f) z, z-x_{n}\right\rangle \leq 0
$$

Indeed, we can choose a subsequence $\left\{v_{n_{i}}\right\}$ of $\left\{v_{n}\right\}$ such that

$$
\lim _{i \rightarrow \infty}\left\langle(B-\gamma f) z, z-v_{n_{i}}\right\rangle=\limsup _{n \rightarrow \infty}\left\langle(B-\gamma f) z, z-v_{n}\right\rangle
$$

Since $\left\{v_{n_{i}}\right\}$ is bounded, there exists a subsequence $\left\{v_{n_{i_{j}}}\right\}$ of $\left\{v_{n_{i}}\right\}$ which converges weakly to $v \in C$. Without loss of generality, we can assume that $v_{n_{i}} \rightarrow v$. From $\left\|W_{n} v_{n}-v_{n}\right\| \rightarrow 0$, we obtain $W_{n} v_{n_{i}} \rightarrow v$. Let us show that $v \in \operatorname{MEP}(F, \varphi)$. Since $u_{n}=T_{r_{n}} x_{n} \in \operatorname{dom} \varphi$, we have

$$
F\left(u_{n}, y\right)+\varphi(y)-\varphi\left(u_{n}\right)+\frac{1}{r_{n}}\left\langle y-u_{n}, u_{n}-x_{n}\right\rangle \geq 0, \quad \forall y \in C
$$

From (A2), we also have

$$
\varphi(y)-\varphi\left(u_{n}\right)+\frac{1}{r_{n}}\left\langle y-u_{n}, u_{n}-x_{n}\right\rangle \geq F\left(y, u_{n}\right), \quad \forall y \in C,
$$

and hence

$$
\varphi(y)-\varphi\left(u_{n}\right)+\left\langle y-u_{n_{i}}, \frac{u_{n_{i}}-x_{n_{i}}}{r_{n_{i}}}\right\rangle \geq F\left(y, u_{n_{i}}\right), \quad \forall y \in C
$$

From $\left\|x_{n}-u_{n}\right\| \rightarrow 0,\left\|x_{n}-W_{n} v_{n}\right\| \rightarrow 0$, and $\left\|W_{n} v_{n}-v_{n}\right\| \rightarrow 0$, we get $u_{n_{i}} \rightarrow v$. Since $\left(u_{n_{i}}-x_{n_{i}}\right) / r_{n_{i}} \rightarrow 0$, it follows by (A4) and the weakly lower semicontinuity of $\varphi$ that

$$
F(y, v)+\varphi(v)-\varphi(y) \leq 0, \quad \forall y \in C
$$

For $t$ with $0<t \leq 1$ and $y \in C$, let $y_{t}=t y+(1-t) v$. Since $y \in C$ and $v \in C$, we have $y_{t} \in C$, and hence $F\left(y_{t}, v\right)+\varphi(v)-\varphi\left(y_{t}\right) \leq 0$. So, from (A1), (A4), and the convexity of $\varphi$, we have

$$
\begin{aligned}
0 & =F\left(y_{t}, y_{t}\right)+\varphi\left(y_{t}\right)-\varphi\left(y_{t}\right) \\
& \leq t F\left(y_{t}, y\right)+(1-t) F\left(y_{t}, v\right)+t \varphi(y)+(1-t) \varphi(v)-\varphi\left(y_{t}\right) \\
& \leq t\left(F\left(y_{t}, y\right)+\varphi(y)-\varphi\left(y_{t}\right)\right) .
\end{aligned}
$$

Dividing by $t$, we get $F\left(y_{t}, y\right)+\varphi(y)-\varphi\left(y_{t}\right) \geq 0$. From (A3) and the weakly lower semicontinuity of $\varphi$, we have $F(v, y)+\varphi(y)-\varphi(v) \geq 0$ for all $y \in C$, and hence $v \in \operatorname{MEP}(F, \varphi)$. 
Next, we show that $v \in F\left(W_{n}\right)=\bigcap_{n=1}^{N} F\left(T_{i}\right)$. Assume that $v \notin \bigcap_{n=1}^{N} F\left(T_{i}\right)$. Since $v_{n_{i}} \rightarrow v$ and $W_{n} v \neq v$, from Opial's condition, we have

$$
\begin{aligned}
\liminf _{i \rightarrow \infty}\left\|v_{n_{i}}-v\right\| & <\liminf _{i \rightarrow \infty}\left\|v_{n_{i}}-W_{n} v\right\| \\
& \leq \liminf _{i \rightarrow \infty}\left(\left\|v_{n_{i}}-W_{n} v_{n_{i}}\right\|+\left\|W_{n} v_{n_{i}}-W_{n} v\right\|\right) \\
& \leq \liminf _{i \rightarrow \infty}\left\|v_{n_{i}}-v\right\|,
\end{aligned}
$$

which is a contradiction. Thus, we obtain $v \in F\left(W_{n}\right)=\bigcap_{n=1}^{N} F\left(T_{i}\right)$.

Next, we show that $v \in I(A, M)$. In fact, having $A$ as $\beta$-inverse-strongly monotone, implies that $A$ is $(1 / \beta)$-Lipschitz continuous monotone mapping and that domain of $A$ is equal to $H$. It follows from Lemma 2.7 that $M+A$ is a maximal monotone. Let $(y, g) \in G(M+$ $A)$, that is, $g-A y \in M(y)$. Since $v_{n_{i}}=J_{M, \lambda}\left(y_{n_{i}}-\lambda A y_{n_{i}}\right)$, we have $y_{n_{i}}-\lambda A y_{n_{i}} \in(I+\lambda M)\left(v_{n_{i}}\right)$, that is,

$$
\frac{1}{\lambda}\left(y_{n_{i}}-v_{n_{i}}-\lambda A y_{n_{i}}\right) \in M\left(v_{n_{i}}\right)
$$

With $M+A$ being a maximal monotone, we have

$$
\left\langle y-v_{n_{i}}, g-A y-\frac{1}{\lambda}\left(y_{n_{i}}-v_{n_{i}}-\lambda A y_{n_{i}}\right)\right\rangle \geq 0
$$

and so

$$
\begin{aligned}
\left\langle y-v_{n_{i}}, g\right\rangle & \geq\left\langle y-v_{n_{i}}, A y+\frac{1}{\lambda}\left(y_{n_{i}}-v_{n_{i}}-\lambda A y_{n_{i}}\right)\right\rangle \\
& =\left\langle y-v_{n_{i}}, A y-A v_{n_{i}}+A v_{n_{i}}-A y_{n_{i}}+\frac{1}{\lambda}\left(y_{n_{i}}-v_{n_{i}}\right)\right\rangle \\
& \geq 0+\left\langle y-v_{n_{i}}, A v_{n_{i}}-A y_{n_{i}}\right\rangle+\left\langle y-v_{n_{i}}, \frac{1}{\lambda}\left(y_{n_{i}}-v_{n_{i}}\right)\right\rangle
\end{aligned}
$$

It follows from $\left\|y_{n}-v_{n}\right\| \rightarrow 0,\left\|A y_{n}-A v_{n}\right\| \rightarrow 0$, and $v_{n_{i}} \rightarrow v$ that

$$
\lim _{i \rightarrow \infty}\left\langle y-v_{n_{i}}, g\right\rangle=\langle y-v, g\rangle \geq 0 .
$$

It follows from the maximal monotonicity of $M+A$ that $0 \in(M+A)(v)$, that is, $v \in I(A, M)$. This implies that $v \in \Omega$.

Since $z=P_{\Omega}(I-B+\gamma f)(z)$, it follows that

$$
\begin{aligned}
\limsup _{n \rightarrow \infty}\left\langle(B-\gamma f) z, z-x_{n}\right\rangle & =\limsup _{n \rightarrow \infty}\left\langle(B-\gamma f) z, z-v_{n}\right\rangle \\
& =\lim _{i \rightarrow \infty}\left\langle(B-\gamma f) z, z-v_{n_{i}}\right\rangle=\langle(B-\gamma f) z, z-v\rangle \leq 0 .
\end{aligned}
$$


Journal of Inequalities and Applications

By (3.49), (3.50), and the last inequality, we have

$$
\limsup _{n \rightarrow \infty}\left\langle\gamma f z-B z, W_{n} v_{n}-z\right\rangle \leq 0
$$

Finally, we show that $\left\{x_{n}\right\}$ converges strongly to $z$. Indeed, from (3.1), we have

$$
\begin{aligned}
\left\|x_{n+1}-z\right\|^{2}= & \left\|\alpha_{n} \gamma f\left(x_{n}\right)+\beta_{n} x_{n}+\left(\left(1-\beta_{n}\right) I-\alpha_{n} B\right) W_{n} v_{n}-z\right\|^{2} \\
= & \left\|\alpha_{n}\left(\gamma f\left(x_{n}\right)-B z\right)+\beta_{n}\left(x_{n}-z\right)+\left(\left(1-\beta_{n}\right) I-\alpha_{n} B\right)\left(W_{n} v_{n}-z\right)\right\|^{2} \\
= & \alpha_{n}^{2}\left\|\gamma f\left(x_{n}\right)-B z\right\|^{2}+\left\|\beta_{n}\left(x_{n}-z\right)+\left(\left(1-\beta_{n}\right) I-\alpha_{n} B\right)\left(W_{n} v_{n}-z\right)\right\|^{2} \\
& +2\left\langle\beta_{n}\left(x_{n}-z\right)+\left(\left(1-\beta_{n}\right) I-\alpha_{n} B\right)\left(W_{n} v_{n}-z\right), \alpha_{n}\left(\gamma f\left(x_{n}\right)-B z\right)\right\rangle \\
\leq & \alpha_{n}^{2}\left\|\gamma f\left(x_{n}\right)-B z\right\|^{2}+\left(\beta_{n}\left\|x_{n}-z\right\|+\left(1-\beta_{n}-\alpha_{n} \bar{\gamma}\right)\left\|v_{n}-z\right\|\right)^{2} \\
& +2 \alpha_{n} \beta_{n}\left\langle x_{n}-z, \gamma f\left(x_{n}\right)-B z\right\rangle+2 \alpha_{n}\left(1-\beta_{n}-\alpha_{n} \bar{\gamma}\right)\left\langle W_{n} v_{n}-z, \gamma f\left(x_{n}\right)-B z\right\rangle \\
\leq & \alpha_{n}^{2}\left\|\gamma f\left(x_{n}\right)-B z\right\|^{2}+\left(\beta_{n}\left\|x_{n}-z\right\|+\left(1-\beta_{n}-\alpha_{n} \bar{\gamma}\right)\left\|x_{n}-z\right\|\right)^{2} \\
& +2 \alpha_{n} \beta_{n}\left\langle x_{n}-z, \gamma f\left(x_{n}\right)-\gamma f(z)\right\rangle+2 \alpha_{n} \beta_{n}\left\langle x_{n}-z, \gamma f(z)-B z\right\rangle \\
& +2 \alpha_{n}\left(1-\beta_{n}-\alpha_{n} \bar{\gamma}\right)\left\langle W_{n} v_{n}-z, \gamma f\left(x_{n}\right)-\gamma f(z)\right\rangle \\
& +2 \alpha_{n}\left(1-\beta_{n}-\alpha_{n} \bar{\gamma}\right)\left\langle W_{n} v_{n}-z, \gamma f(z)-B z\right\rangle \\
\leq & \alpha_{n}^{2}\left\|\gamma f\left(x_{n}\right)-B z\right\|^{2}+\left(1-\alpha_{n} \bar{\gamma}\right)^{2}\left\|x_{n}-z\right\|^{2} \\
& +2 \alpha_{n} \beta_{n} \gamma\left\|x_{n}-z\right\|\left\|f\left(x_{n}\right)-f(z)\right\|+2 \alpha_{n} \beta_{n}\left\langle x_{n}-z, \gamma f(z)-B z\right\rangle \\
& +2 \alpha_{n}\left(1-\beta_{n}-\alpha_{n} \bar{\gamma}\right) \gamma\left\|W_{n} v_{n}-z\right\|\left\|f\left(x_{n}\right)-f(z)\right\| \\
& +2 \alpha_{n}\left(1-\beta_{n}-\alpha_{n} \bar{\gamma}\right)\left\langle W_{n} v_{n}-z, \gamma f(z)-B z\right\rangle \\
\leq & \alpha_{n}^{2}\left\|\gamma f\left(x_{n}\right)-B z\right\|^{2}+\left(1-\alpha_{n} \bar{\gamma}\right)^{2}\left\|x_{n}-z\right\|^{2} \\
& +2 \alpha_{n} \beta_{n} \gamma \alpha\left\|x_{n}-z\right\|^{2}+2 \alpha_{n} \beta_{n}\left\langle x_{n}-z, \gamma f(z)-B z\right\rangle \\
& +2 \alpha_{n}\left(1-\beta_{n}-\alpha_{n} \bar{\gamma}\right) \gamma \alpha\left\|x_{n}-z\right\|^{2}+2 \alpha_{n}\left(1-\beta_{n}-\alpha_{n} \bar{\gamma}\right)\left\langle W_{n} v_{n}-z, \gamma f(z)-B z\right\rangle \\
= & \alpha_{n}^{2}\left\|\gamma f\left(x_{n}\right)-B z\right\|^{2}+\left(1-2 \alpha_{n} \bar{\gamma}+\alpha_{n}^{2} \bar{\gamma}^{2}+2 \alpha_{n} \gamma \alpha-2 \alpha_{n}^{2} \bar{\gamma} \gamma \alpha\right)\left\|x_{n}-z\right\|^{2} \\
& +2 \alpha_{n} \beta_{n}\left\langle x_{n}-z, \gamma f(z)-B z\right\rangle+2 \alpha_{n}\left(1-\beta_{n}-\alpha_{n} \bar{\gamma}\right)\left\langle W_{n} v_{n}-z, \gamma f(z)-B z\right\rangle \\
= & \left(1-\alpha_{n}\left(2 \bar{\gamma}-\alpha_{n} \bar{\gamma}^{2}-2 \gamma \alpha+2 \alpha_{n} \bar{\gamma} \gamma \alpha\right)\right)\left\|x_{n}-z\right\|^{2}+\alpha_{n} \sigma_{n}, \\
&
\end{aligned}
$$

where $\sigma_{n}=\alpha_{n}\left\|\gamma f\left(x_{n}\right)-B z\right\|^{2}+2 \beta_{n}\left\langle x_{n}-z, \gamma f(z)-B z\right\rangle+2\left(1-\beta_{n}-\alpha_{n} \bar{\gamma}\right)\left\langle W_{n} v_{n}-z, \gamma f(z)-B z\right\rangle$. By (3.65), we get lim $\sup _{n \rightarrow \infty} \sigma_{n} \leq 0$. Hence by Lemma 2.2 to (3.66), we conclude that $x_{n} \rightarrow z$. This completes the proof. 
Using Theorem 3.1, we obtain the following corollaries.

Corollary 3.2. Let $C$ be a nonempty closed convex subset of a real Hilbert Space $H$. Let $F$ be a bifunction of $C \times C$ into real numbers $\mathbb{R}$ satisfying (A1)-(A5) and let $f$ be a contraction of $H$ into itself with coefficient $\alpha \in(0,1)$. Let $A$ be an $\beta$-inverse-strongly monotone mapping of $H$ into itself and let $M: H \rightarrow 2^{H}$ be a maximal monotone mapping such that $\Theta:=F(T) \cap I(A, M) \cap \operatorname{EP}(F) \neq \emptyset$. Let $\left\{x_{n}\right\}$ be a sequence generated by $x_{1} \in H$ and

$$
\begin{gathered}
F\left(u_{n}, y\right)+\frac{1}{r_{n}}\left\langle y-u_{n}, u_{n}-x_{n}\right\rangle \geq 0, \quad \forall y \in C, \\
y_{n}=J_{M, \lambda}\left(u_{n}-\lambda A u_{n}\right), \\
x_{n+1}=\alpha_{n} \gamma f\left(x_{n}\right)+\beta_{n} x_{n}+\left(1-\beta_{n}-\alpha_{n}\right) T J_{M, \lambda}\left(y_{n}-\lambda A y_{n}\right)
\end{gathered}
$$

for every $n \geq 1$, where $\left\{\alpha_{n}\right\},\left\{\beta_{n}\right\} \subset(0,1),\left\{r_{n}\right\} \subset(0, \infty)$, and $\lambda \in(0,2 \beta)$ satisfy the conditions (i)-(iii) in Theorem 3.1. Then $\left\{x_{n}\right\}$ converges strongly to $z=P_{\Theta} \gamma f(z)$.

Proof. Taking $T_{i}=T$ for $i=1,2, \ldots, N, B=I$, and $\varphi=0$, in Theorem 3.1, we can conclude the desired conclusion easily. This completes the proof.

Corollary 3.3. Let $C$ be a nonempty closed convex subset of a real Hilbert Space $H$. Let $F$ be a bifunction of $C \times C$ into real numbers $\mathbb{R}$ satisfying (A1)-(A5) and let $\varphi: C \rightarrow \mathbb{R} \cup\{+\infty\}$ be a proper lower semicontinuous and convex function. Let $f$ be a contraction of $H$ into itself with coefficient $\alpha \in(0,1)$. Let $A$ be an $\beta$-inverse-strongly monotone mapping of $C$ into $H$ and let $B$ be a strongly bounded linear operator on $H$ with coefficient $\bar{\gamma}>0$ and $0<\gamma<\bar{\gamma} / \alpha$. Let $T_{1}, T_{2}, \ldots, T_{N}$ be a family of finitely nonexpansive mappings of $C$ into $H$ such that $\Upsilon:=\bigcap_{n=1}^{N} F\left(T_{i}\right) \cap \operatorname{VI}(C, A) \cap \operatorname{MEP}(F, \varphi) \neq \emptyset$ and let $W_{n}$ be the $W$-mapping generated by $T_{1}, T_{2}, \ldots, T_{N}$ and $\lambda_{n, 1}, \lambda_{n, 2}, \ldots, \lambda_{n, N}$. Assume that either (B1) or (B2) holds. Let $\left\{x_{n}\right\}$ be a sequence generated by $x_{1} \in H$ and

$$
\begin{gathered}
F\left(u_{n}, y\right)+\varphi(y)-\varphi\left(u_{n}\right)+\frac{1}{r_{n}}\left\langle y-u_{n}, u_{n}-x_{n}\right\rangle \geq 0, \quad \forall y \in C, \\
y_{n}=P_{C}\left(u_{n}-\lambda A u_{n}\right), \\
v_{n}=P_{C}\left(y_{n}-\lambda A y_{n}\right), \\
x_{n+1}=\alpha_{n} \gamma f\left(x_{n}\right)+\beta_{n} x_{n}+\left(\left(1-\beta_{n}\right) I-\alpha_{n} B\right) W_{n} v_{n}
\end{gathered}
$$

For every $n \geq 1$. where $\left\{\alpha_{n}\right\},\left\{\beta_{n}\right\} \subset(0,1),\left\{r_{n}\right\} \subset(0, \infty)$, and $\lambda \in(0,2 \beta)$ satisfy the condition (i)-(iv) in Theorem 3.1. Then $\left\{x_{n}\right\}$ converges strongly to $z \in \Upsilon$ which is the unique solution of the variational inequality

$$
\langle(B-\gamma f) z, z-x\rangle \leq 0, \quad x \in \Upsilon
$$

Equivalently, one has $z=P_{\Upsilon}(I-B+\gamma f)(z)$.

Proof. From Theorem 3.1 put $M=\partial \delta_{C}$; then $J_{M, \lambda}=P_{C}$. So we have $y_{n}=P_{C}\left(u_{n}-\lambda A u_{n}\right)$ and $v_{n}=P_{C}\left(y_{n}-\lambda A y_{n}\right)$. The conclusion of Corollary 3.3 can be obtained from Theorem 3.1 immediately. 


\section{Application}

In this section, we study a kind of optimization problem by using the result of this paper. We will give an iterative algorithm of solution for the following optimization problem with nonempty set of solutions:

$$
\min h(x), \quad x \in C,
$$

where $h(x)$ is a convex and lower semicontinuous functional defined convex subset $C$ of a Hilbert space $H$. We denote by $M(h)$ the set of solutions of (4.1). Let $F: C \times C \rightarrow \mathbb{R}$ be a bifunction defined by $F(x, y)=h(y)-h(x)$. We consider the equilibrium problem (1.8); it is obvious that $\operatorname{EP}(F)=\operatorname{Min}(h)$. Therefore, from Theorem 3.1, we give the following corollary.

Corollary 4.1. Let $C$ be a nonempty closed convex subset of a real Hilbert Space $H$. Let $F$ be a bifunction of $C \times C$ into real numbers $\mathbb{R}$ satisfying (A1)-(A5) and let $h: C \rightarrow \mathbb{R} \cup\{+\infty\}$ be a lower semicontinuous and convex function. Let $f$ be a contraction of $H$ into itself with coefficient $\alpha \in(0,1)$. Let $A$ be an $\beta$-inverse-strongly monotone mapping of $H$ into itself, let $M: H \rightarrow 2^{H}$ be a maximal monotone mapping, and let $B$ be a strongly bounded linear operator on $H$ with coefficient $\bar{\gamma}>0$ and $0<\gamma<\bar{\gamma} / \alpha$. Let $T_{1}, T_{2}, \ldots, T_{N}$ be a family of finitely nonexpansive mappings of $C$ into $H$ such that

$\Phi:=\bigcap_{n=1}^{N} F\left(T_{i}\right) \cap I(A, M) \cap \operatorname{Min}(h) \neq \emptyset$ and let $W_{n}$ be the $W$-mapping generated by $T_{1}, T_{2}, \ldots, T_{N}$ and $\lambda_{n, 1}, \lambda_{n, 2}, \ldots, \lambda_{n, N}$. Let $\left\{x_{n}\right\}$ be a sequence generated by $x_{1} \in H$ and

$$
\begin{gathered}
h(y)-h\left(u_{n}\right)+\frac{1}{r_{n}}\left\langle y-u_{n}, u_{n}-x_{n}\right\rangle \geq 0, \quad \forall y \in C, \\
y_{n}=J_{M, \lambda}\left(u_{n}-\lambda A u_{n}\right), \\
v_{n}=J_{M, \lambda}\left(y_{n}-\lambda A y_{n}\right), \\
x_{n+1}=\alpha_{n} \gamma f\left(x_{n}\right)+\beta_{n} x_{n}+\left(\left(1-\beta_{n}\right) I-\alpha_{n} B\right) W_{n} v_{n}
\end{gathered}
$$

for every $n \geq 1$, where $\left\{\alpha_{n}\right\},\left\{\beta_{n}\right\} \subset(0,1),\left\{r_{n}\right\} \subset(0, \infty)$, and $\lambda \in(0,2 \beta)$ satisfy the following conditions:

(i) $\sum_{n=0}^{\infty} \alpha_{n}=\infty$ and $\lim _{n \rightarrow \infty} \alpha_{n}=0$.

(ii) $\liminf _{n \rightarrow \infty} r_{n}>0$ and $\lim _{n \rightarrow \infty}\left|r_{n+1}-r_{n}\right|=0$.

(iii) $0<\liminf _{n \rightarrow \infty} \beta_{n} \leq \lim \sup _{n \rightarrow \infty} \beta_{n}<1$.

(iv) $\lim _{n \rightarrow \infty}\left|\lambda_{n, i}-\lambda_{n-1, i}\right|=0$ for all $i=1,2, \ldots, N$.

Then $\left\{x_{n}\right\}$ converges strongly to $z \in \Phi$, where $z=P_{\Phi}(I-B+\gamma f)(z)$, which is the unique solution of the variational inequality

$$
\langle(B-\gamma f) z, z-x\rangle \leq 0, \quad x \in \Phi
$$

Proof. From Theorem 3.1 put $F\left(u_{n}, y\right)=h(y)-h\left(u_{n}\right)$ and $\varphi \equiv 0$. The conclusion of Corollary 4.1 can be obtained from Theorem 3.1 immediately. 


\section{Acknowledgments}

The authors would like to thank the Centre of Excellence in Mathematics, under the Commission on Higher Education, Ministry of Education, Thailand. Mr. Phayap Katchang was supported by King Mongkut's Diamond scholarship for fostering special academic skills by KMUTT for Ph.D. Program at KMUTT. Moreover, the authors are also very grateful to Professor Yeol Je Cho and Professor Jong Kyu Kim for the hospitality.

\section{References}

[1] M. A. Noor, "Generalized set-valued variational inclusions and resolvent equations," Journal of Mathematical Analysis and Applications, vol. 228, no. 1, pp. 206-220, 1998.

[2] F. E. Browder, "Nonlinear monotone operators and convex sets in Banach spaces," Bulletin of the American Mathematical Society, vol. 71, no. 5, pp. 780-785, 1965.

[3] P. Hartman and G. Stampacchia, "On some non-linear elliptic differential-functional equations," Acta Mathematica, vol. 115, no. 1, pp. 271-310, 1966.

[4] J.-L. Lions and G. Stampacchia, "Variational inequalities," Communications on Pure and Applied Mathematics, vol. 20, pp. 493-519, 1967.

[5] H. Brézis, Opérateurs Maximaux Monotones et Semi-Groupes de Contractions dans Les Espaces de Hilbert, North-Holland Mathematics Studies, no. 5. Notas de Matemática (50), North-Holland, Amsterdam, The Netherlands, 1973.

[6] S.-S. Zhang, J. H. W. Lee, and C. K. Chan, "Algorithms of common solutions to quasi variational inclusion and fixed point problems," Applied Mathematics and Mechanics, vol. 29, no. 5, pp. 571-581, 2008.

[7] L.-C. Ceng and J.-C. Yao, "A hybrid iterative scheme for mixed equilibrium problems and fixed point problems," Journal of Computational and Applied Mathematics, vol. 214, no. 1, pp. 186-201, 2008.

[8] E. Blum and W. Oettli, "From optimization and variational inequalities to equilibrium problems," The Mathematics Student, vol. 63, no. 1-4, pp. 123-145, 1994.

[9] Y. J. Cho, X. Qin, and J. I. Kang, "Convergence theorems based on hybrid methods for generalized equilibrium problems and fixed point problems," Nonlinear Analysis: Theory, Methods E Applications, vol. 71, no. 9, pp. 4203-4214, 2009.

[10] S. D. Flåm and A. S. Antipin, "Equilibrium programming using proximal-like algorithms," Mathematical Programming, vol. 78, no. 1, pp. 29-41, 1997.

[11] C. Jaiboon and P. Kumam, "A hybrid extragradient viscosity approximation method for solving equilibrium problems and fixed point problems of infinitely many nonexpansive mappings," Fixed Point Theory and Applications, vol. 2009, Article ID 374815, 32 pages, 2009.

[12] C. Jaiboon, P. Kumam, and U. W. Humphries, "Weak convergence theorem by an extragradient method for variational inequality, equilibrium and fixed point problems," Bulletin of the Malaysian Mathematical Sciences Society, vol. 32, no. 2, pp. 173-185, 2009.

[13] P. Kumam, "Strong convergence theorems by an extragradient method for solving variational inequalities and equilibrium problems in a Hilbert space," Turkish Journal of Mathematics, vol. 33, no. 1, pp. 85-98, 2009.

[14] P. Kumam, "A hybrid approximation method for equilibrium and fixed point problems for a monotone mapping and a nonexpansive mapping," Nonlinear Analysis: Hybrid Systems, vol. 2, no. 4, pp. 1245-1255, 2008.

[15] P. Kumam, "A new hybrid iterative method for solution of equilibrium problems and fixed point problems for an inverse strongly monotone operator and a nonexpansive mapping," Journal of Applied Mathematics and Computing, vol. 29, no. 1-2, pp. 263-280, 2009.

[16] P. Kumam and P. Katchang, "A viscosity of extragradient approximation method for finding equilibrium problems, variational inequalities and fixed point problems for nonexpansive mappings," Nonlinear Analysis: Hybrid Systems, vol. 3, no. 4, pp. 475-486, 2009.

[17] P. Katchang and P. Kumam, "A new iterative algorithm of solution for equilibrium problems, variational inequalities and fixed point problems in a Hilbert space," Journal of Applied Mathematics and Computing, vol. 32, no. 1, pp. 19-38, 2010.

[18] A. Moudafi and M. Théra, "Proximal and dynamical approaches to equilibrium problems," in Ill-Posed Variational Problems and Regularization Techniques (Trier, 1998), vol. 477 of Lecture Notes in Economics and Mathematical Systems, pp. 187-201, Springer, Berlin, Germany, 1999. 
[19] X. Qin, Y. J. Cho, and S. M. Kang, "Viscosity approximation methods for generalized equilibrium problems and fixed point problems with applications," Nonlinear Analysis: Theory, Methods $\mathcal{E}$ Applications, vol. 72, no. 1, pp. 99-112, 2010.

[20] X. Qin, Y. J. Cho, and S. M. Kang, "Convergence theorems of common elements for equilibrium problems and fixed point problems in Banach spaces," Journal of Computational and Applied Mathematics, vol. 225, no. 1, pp. 20-30, 2009.

[21] Y. Yao, Y. J. Cho, and R. Chen, "An iterative algorithm for solving fixed point problems, variational inequality problems and mixed equilibrium problems," Nonlinear Analysis: Theory, Methods $\mathcal{E}$ Applications, vol. 71, no. 7-8, pp. 3363-3373, 2009.

[22] J.-W. Peng, Y. Wang, D. S. Shyu, and J.-C. Yao, "Common solutions of an iterative scheme for variational inclusions, equilibrium problems, and fixed point problems," Journal of Inequalities and Applications, vol. 2008, Article ID 720371, 15 pages, 2008.

[23] S. Plubtieng and W. Sriprad, "A viscosity approximation method for finding common solutions of variational inclusions, equilibrium problems, and fixed point problems in Hilbert spaces," Fixed Point Theory and Applications, vol. 2009, Article ID 567147, 20 pages, 2009.

[24] S. Atsushiba and W. Takahashi, "Strong convergence theorems for a finite family of nonexpansive mappings and applications," Indian Journal of Mathematics, vol. 41, no. 3, pp. 435-453, 1999.

[25] W. Takahashi and K. Shimoji, "Convergence theorems for nonexpansive mappings and feasibility problems," Mathematical and Computer Modelling, vol. 32, no. 11-13, pp. 1463-1471, 2000.

[26] W. Takahashi, "Weak and strong convergence theorems for families of nonexpansive mappings and their applications," Annales Universitatis Mariae Curie-Skłodowska. Sectio A, vol. 51, no. 2, pp. 277-292, 1997.

[27] L. C. Ceng, P. Cubiotti, and J. C. Yao, "Strong convergence theorems for finitely many nonexpansive mappings and applications," Nonlinear Analysis: Theory, Methods E Applications, vol. 67, no. 5, pp. 1464-1473, 2007.

[28] V. Colao, G. Marino, and H.-K. Xu, "An iterative method for finding common solutions of equilibrium and fixed point problems," Journal of Mathematical Analysis and Applications, vol. 344, no. 1, pp. 340 352, 2008.

[29] J.-W. Peng and J.-C. Yao, "Two extragradient methods for generalized mixed equilibrium problems, nonexpansive mappings and monotone mappings," Computers $\mathcal{E}$ Mathematics with Applications, vol. 58, no. 7, pp. 1287-1301, 2009.

[30] Z. Opial, "Weak convergence of the sequence of successive approximations for nonexpansive mappings," Bulletin of the American Mathematical Society, vol. 73, pp. 591-597, 1967.

[31] J.-W. Peng and J.-C. Yao, "Strong convergence theorems of iterative scheme based on the extragradient method for mixed equilibrium problems and fixed point problems," Mathematical and Computer Modelling, vol. 49, no. 9-10, pp. 1816-1828, 2009.

[32] H.-K. Xu, "Viscosity approximation methods for nonexpansive mappings," Journal of Mathematical Analysis and Applications, vol. 298, no. 1, pp. 279-291, 2004.

[33] M. O. Osilike and D. I. Igbokwe, "Weak and strong convergence theorems for fixed points of pseudocontractions and solutions of monotone type operator equations," Computers $\mathcal{E}$ Mathematics with Applications, vol. 40, no. 4-5, pp. 559-567, 2000.

[34] T. Suzuki, "Strong convergence of Krasnoselskii and Mann's type sequences for one-parameter nonexpansive semigroups without Bochner integrals," Journal of Mathematical Analysis and Applications, vol. 305, no. 1, pp. 227-239, 2005.

[35] G. Marino and H.-K. Xu, "A general iterative method for nonexpansive mappings in Hilbert spaces," Journal of Mathematical Analysis and Applications, vol. 318, no. 1, pp. 43-52, 2006. 